BULLETIN OF THE

AMERICAN MATHEMATICAL SOCIETY

Volume 77, Number 2, March 1971

\title{
CHEVALLEY GROUPS OVER COMMUTATIVE RINGS ${ }^{1}$
}

BY MICHAEL R. STEIN ${ }^{2}$

Communicated by Hyman Bass, August 26, 1970

1. Introduction. Steinberg [8] has given a simple presentation for the universal central extension [7], [8], [9] of the group of rational points of a simply connected Chevalley group over a field. In this note we announce a similar theory for the simply connected Chevalley groups over commutative rings and outline the proof of a stability theorem for certain functors resulting from this construction. Complete proofs will appear elsewhere.

Let us introduce some notation. $A$ denotes a commutative ring with $1, A^{*}$ is its group of invertible elements, $\mathfrak{p}$ and $\mathfrak{q}$ are ideals of $A$, and $(1+\mathfrak{q})^{*}=(1+\mathfrak{q}) \cap A^{*} . \Phi$ is a reduced irreducible root system [2] and $G(\Phi, \quad)$ is the simply connected Chevalley-Demazure group scheme with root system $\Phi$. If $\Phi$ is of type $C_{l}, l \geqq 1\left(C_{1}=A_{1}\right)$, we say $\Phi$ is symplectic, and if $\Phi$ is of type $A_{l}, B_{l}, C_{l}$, or $D_{l}$, we say $\Phi$ is classical. The subgroup of $G(\Phi, A)$ generated by the elementary unipotents $e_{\alpha}(t), \alpha \in \Phi, t \in A$, will be denoted $E(\Phi, A)$. A full discussion of these notions may be found in [3], [5], and [9].

Define the Steinberg group, $\operatorname{St}(\Phi, A)$, to be the group with generators $x_{\alpha}(t), \alpha \in \Phi, t \in A$, subject to the relations

$$
\begin{aligned}
x_{\alpha}(s) x_{\alpha}(t) & =x_{\alpha}(s+t) & & (\alpha \in \Phi ; s, t \in A) \\
{\left[x_{\alpha}(s), x_{\beta}(t)\right] } & =\prod x_{i \alpha+j \beta}\left(N_{\alpha, \beta, i, j} s^{i} t^{j}\right) & & (\alpha, \beta \in \Phi, \alpha+\beta \neq 0)
\end{aligned}
$$

where the product is as in [8]. Since the elementary unipotents $e_{\alpha}(t)$ also satisfy these relations, the map $x_{\alpha}(t) \mapsto e_{\alpha}(t)$ extends to a homomorphism $\pi: \operatorname{St}(\Phi, A) \rightarrow G(\Phi, A)$ with image $E(\Phi, A)$. Set ker $\pi=L(\Phi, A)$.

In $\$ 2$ we present certain commutator formulas which yield necessary and sufficient conditions for $E(\Phi, A)$ and $\operatorname{St}(\Phi, A)$ to be their own derived groups. In $\$ 3$ we show that the extension $\operatorname{St}(\Phi, A) \rightarrow E(\Phi, A)$

AMS 1970 subject classifications. Primary 18F25, 20G10, 20G35; Secondary 20G25, 20G30.

Key words and phrases. Chevalley group, universal central extension, stability theorems, Steinberg group, commutators in Chevalley groups, $K_{2}$, second homology group, Bruhat decomposition.

1 This paper is a portion of the author's doctoral thesis at Columbia University, written under the direction of Professor Hyman Bass. The research was supported in part by National Science Foundation Grants NSF-GP-8718 and NSF-GP-13711.

2 Present address: Northwestern University, Evanston, Illinois 60201. 
is "stably central," and, under certain restrictions on $\mathrm{rk} \Phi$, that every central extension of $\operatorname{St}(\Phi, A)$ splits. This generalizes results of Milnor [6], Kervaire [4] and Steinberg [9] for $\operatorname{SL}(n, A), n \geqq 3$ (the Chevalley groups of type $\left.A_{n}, n \geqq 2\right)$. The functor $\lim _{n \rightarrow \infty} L\left(A_{n}, \quad\right)$ is Milnor's algebraic $K_{2}$ functor, and similar functors arise from the results of $\$ 2$ and $\$ 3$ for the other Chevalley groups.

Now suppose $\Phi_{l}$ is a root system of rank $l$. An inclusion $\Phi_{l} \subset \Phi_{l+1}$ induces homomorphisms of the corresponding groups and, in particular, a map $\theta_{l}: L\left(\Phi_{l}, A\right) \rightarrow L\left(\Phi_{l+1}, A\right)$. When $A$ is a field, Steinberg [8] has shown that the maps $\theta_{l}$ are always surjective. This partially answers a stability question: how large must $l$ be, relative to the dimension of the maximal ideal space of $A$, for $\theta_{l}$ to be surjective? In $\S 4$ we show that $l=1$ suffices for almost all semilocal rings-rings with $\operatorname{dim} \max A$ equal to 0 . Consequences of this result will be discussed in $\$ 4$.

\section{Commutators in Chevalley groups. Set}

$$
\operatorname{St}(\Phi, \mathfrak{q})=\operatorname{ker}(\operatorname{St}(\Phi, A) \rightarrow \operatorname{St}(\Phi, A / \mathfrak{q})) .
$$

The image of $\operatorname{St}(\Phi, \mathfrak{q})$ under $\pi$ is denoted $E(\Phi, \mathfrak{q})$, and $L(\Phi, \mathfrak{q})$ $=\operatorname{ker} \pi \cap \operatorname{St}(\Phi, \mathfrak{q})$. We let $E(\mathfrak{p}, \mathfrak{q})$ be the smallest normal subgroup of $E(\Phi, A)$ containing $\left\{e_{\alpha}(p) \mid p \in \mathfrak{p}, \alpha\right.$ short $\} \cup\left\{e_{\beta}(q) \mid q \in \mathfrak{q}, \beta\right.$ long $\}$. If $\Phi$ has only one root length, by convention all roots are long.

(2.1) $[E(\mathfrak{q}), \quad E(\mathfrak{p})] \supset E\left((u-1) \mathfrak{p}, \quad\left(u^{n}-1\right) \mathfrak{p}\right)$ for all $u \in(1+\mathfrak{q})^{*}$, where $n=1$ if $\Phi$ is nonsymplectic and $n=2$ if $\Phi$ is symplectic.

(2.2) Let $d[q]$ be the ideal generated by $\left\{q^{2}-q \mid q \in \mathfrak{q}\right\}$ and let $s[\mathfrak{q}]$ be the ideal generated by $\left\{q^{2} \mid q \in \mathfrak{q}\right\}$. It has been shown by Bass and Tate $[1]$ that $d[A]=\cap \mathfrak{m}$, where $\mathfrak{m}$ ranges over all maximal ideals of $A$ such that $A / \mathfrak{m} \approx F_{2}$. Moreover, $d[A] \mathfrak{q} \subset d[\mathfrak{q}] \subset \mathfrak{q}$.

Let $F$ be a subgroup of $G(\Phi, A)$ normalized by $E(\Phi, A)$ and, set $F^{\prime}=[E(\mathfrak{q}), F]$.

(2.3) Theorem. Assume $\mathrm{rk} \Phi \geqq 2$, and suppose for some $\gamma \in \Phi$ and some $q_{0} \in A$ that $e_{\gamma}\left(q_{0}\right) \in F$. Then $E(\mathfrak{a}, \mathfrak{b}) \subset F^{\prime}$, where $\mathfrak{a}=\mathfrak{b}=\mathfrak{q} q_{0}$, except in the following cases:

$$
\begin{array}{rlrl}
\gamma \text { long: } \Phi=G_{2} & & \mathfrak{a}=d[\mathfrak{q}] q_{0} \\
\Phi & =C_{2} & & \mathfrak{a}=d[\mathfrak{q}] q_{0}+\mathfrak{q} d\left[A q_{0}\right] \\
& \mathfrak{b}=d[\mathfrak{q}] q_{0}^{2}+\mathfrak{q} d\left[A q_{0}\right] q_{0}+2 \mathfrak{q} q_{0} \\
\Phi=C_{l}, \quad l>2 & \mathfrak{b}=\mathfrak{q} q_{0}^{2}+2 \mathfrak{q} q_{0}+s[\mathfrak{q}] q_{0} \\
\gamma \text { short: } \Phi=G_{2} & \mathfrak{a}=2 \mathfrak{q} q_{0}+d[\mathfrak{q}] q_{0}+3 d[\mathfrak{q}] q_{0} \\
& & \mathfrak{b}=\mathfrak{q} q_{0}^{3}+3 \mathfrak{q} q_{0}
\end{array}
$$




$$
\begin{array}{ll}
\Phi=C_{2} & \mathfrak{a}=d[\mathfrak{q}] q_{0}+\mathfrak{q} d\left[A q_{0}\right] \\
& \mathfrak{b}=d[\mathfrak{q}] q_{0}^{2}+\mathfrak{q} d\left[A q_{0}\right] q_{0}+2 \mathfrak{q} q_{0} \\
\Phi=B_{l}, C_{l}, F_{4}, \quad l>2 & \mathfrak{b}=\mathfrak{q} q_{0}^{2}+2 \mathfrak{q} q_{0} .
\end{array}
$$

Moreover, if $A$ has no residue field with two elements, the case $\Phi=C_{2}$ is the same as $\Phi=C_{l}, l>2$ when $\gamma$ is long.

(2.4) Corollary. $[E(\Phi, A), E(\Phi, \mathfrak{q})]=E(\Phi, \mathfrak{q})$ provided, when $\Phi=C_{2}$ or $G_{2}$, that $A$ has no residue field with two elements, and when $\Phi=A_{1}$, that the elements $u^{2}-1, u \in A^{*}$, generate the unit ideal of $A$.

The case of $A_{1}$ follows from (2.1); the others from (2.2) and (2.3), with $F=E(A), q_{0}=1$.

Remarks. (a) For $q=A$, the hypotheses of (2.4) are necessary and sufficient. To see this, note that $E(A) \rightarrow E(A / \mathfrak{p})$ is surjective, which shows that $E(A / \mathfrak{p})$ is its own derived group whenever $E(A)$ is. However, it is well known that the groups $\operatorname{SL}\left(2, F_{2}\right), \operatorname{SL}\left(2, F_{3}\right), \operatorname{Sp}\left(4, F_{2}\right)$, and $G_{2}\left(F_{2}\right)$ contain normal subgroups of index 2 .

(b) (2.1), (2.3), and (2.4) remain true if $E$ is replaced by $\mathrm{St}$ throughout and $F$ is taken to be a normal subgroup of $\operatorname{St}(\Phi, A)$.

3. Central extensions and $H_{2}$. Recall [7], [9] that the universal central extension of a group $G$ is a central extension, $\hat{G}$, of $G$ which is its own derived group and which has no nonsplit central extensions of its own. These conditions characterize $\hat{G}$ up to unique isomorphism, and $\operatorname{ker}(\hat{G} \rightarrow G) \approx H_{2}(G, \boldsymbol{Z})$.

(3.1) TheOREM. Let $\Phi$ be a reduced irreducible root system, of rank $\geqq 5$ if $\Phi$ is of type $B_{l}$ or $D_{l}$, and of rank $\geqq 4$ otherwise. Then $\operatorname{St}(\Phi, A)$ has no nonsplit central extensions. If $A$ has no residue field with two elements, the same is true for $\Phi=C_{3}$ or $B_{4}$. If the elements $u^{2}-1, u \in A^{*}$, generate the unit ideal of $A$, then $\operatorname{St}(\Phi, A)$ has no nonsplit central extensions whenever $\mathrm{rk} \Phi \geqq 3$.

Outline of Proof. Given a central extension $p: F \rightarrow \operatorname{St}(\Phi, A)$, we must construct a section $s: \operatorname{St}(\Phi, A) \rightarrow F$ of $p$. Over each subgroup of type $A_{2}, B_{3}$, and $C_{3}$ we define canonical liftings, $y_{\alpha}(t)$, of the generators $x_{\alpha}(t)$ of $\operatorname{St}(\Phi, A)$ belonging to that subgroup and then prove that the liftings so defined are independent of the subgroup chosen. Finally we verify that relations (1.1) hold for the elements $y_{\alpha}(t)$, showing that $x_{\alpha}(t) \mapsto y_{\alpha}(t)$ defines a homomorphism which is the desired section for $p$. Each step involves technical considerations which differ from root system to root system, accounting for the rather complicated hypotheses of (3.1).

REMARK. For SL $(n, A),(3.1)$ is due independently to Steinberg [9] 
and Kervaire [4]. It is possible to weaken the hypotheses on $A$ slightly for the cases $\Phi=A_{3}, B_{3}, D_{4}$ (e.g. $D_{4}\left(F_{3}\right)$ has no nonsplit central extensions). If $A$ has enough units, the theorem may be extended to groups of rank $<3$ using the method of [8].

(3.2) Theorem. Let $\Phi_{0}$ be a simple system in $\Phi$ [3], let $\alpha \in \Phi_{0}$, and denote by $\Phi^{\prime}$ the subsystem of $\Phi$ generated by $\Phi_{0}-\{\alpha\}$. Then $\operatorname{ker} \pi \cap M$ is central in $\operatorname{St}(\Phi, A)$, where $M$ is the image of $\operatorname{St}\left(\Phi^{\prime}, A\right)$ in $\operatorname{St}(\Phi, A)$ under the map induced by $\Phi^{\prime} \subset \Phi$.

Corollary. If $\Phi, A$ are as in (3.1), then $\operatorname{St}(\Phi, A) /[\operatorname{ker} \pi, \operatorname{St}(\Phi, A)]$ is the universal central extension of $E(\Phi, A)$. In particular, whenever $\pi$ is central, $L(\Phi, A) \approx H_{2}(E(\Phi, A), \boldsymbol{Z})$.

If $\Phi$ is classical, let $\operatorname{St}_{\infty}(\Phi, A), E_{\infty}(\Phi, A), L_{\infty}(\Phi, A)$ be the direct limits as $l \rightarrow \infty$ of the groups $\operatorname{St}\left(\Phi_{l}, A\right), E\left(\Phi_{l}, A\right), L\left(\Phi_{l}, A\right)$.

(3.3) Corollary. If $\Phi$ is classical, then $\operatorname{St}_{\infty}(\Phi, A)$ is the universal central extension of $E_{\infty}(\Phi, A)$ and $L_{\infty}(\Phi, A) \approx H_{2}\left(E_{\infty}(\Phi, A), Z\right)$.

4. Stability in dimension 0 . For $u \in A^{*}, \alpha \in \Phi$, define elements $\hat{h}_{\alpha}(u)$ as in [8]. Let $\hat{H}(\Phi, A)$ be the subgroup of $\operatorname{St}(\Phi, A)$ generated by the $\hat{h}_{\alpha}(u)$, and let $\hat{H}(\Phi, q)$ be the smallest normal subgroup of $\hat{H}(\Phi, A)$ containing all $\hat{h}_{\alpha}(v), v \in(1+q)^{*}, \alpha \in \Phi$.

The pairing $(u, v) \mapsto\{u, v\}_{l}=\hat{h}_{\alpha}(u v) \hat{h}_{\alpha}(u)^{-1} \hat{h}_{\alpha}(v)^{-1}$ takes values in $L\left(\Phi_{l}, \mathfrak{q}\right)$ if $u$ or $v$ is in $(1+q)^{*}$ and is independent of the long root $\alpha$ chosen. Denote the subgroup of $L\left(\Phi_{l}, \mathfrak{q}\right)$ generated by the values of $\{,\}_{l}$ by $D\left(\Phi_{l}, \mathfrak{q}\right) . D\left(\Phi_{l}, \mathrm{q}\right)$ is a central subgroup of $\operatorname{St}\left(\Phi_{l}, A\right)$ (cf. [9]), and the induced map $D\left(\Phi_{l}, \mathfrak{q}\right) \rightarrow D\left(\Phi_{l+1}, \mathfrak{q}\right)$ is clearly surjective.

If $S$ is a subset of $A$, we write $Z[S]$ for the subring of $A$ generated by $S$.

(4.1) Theorem. Let $\mathfrak{q}$ be an ideal contained in $\operatorname{rad} A$. If $\Phi$ is symplectic assume $A=Z\left[\left(A^{*}\right)^{2}\right]$; otherwise assume $A=Z\left[A^{*}\right]$. Then $L(\Phi, \mathfrak{q})=D(\Phi, \mathfrak{q})$. In particular, the restrictions of the maps $\theta_{l}$ of $\$ 1$ to $L\left(\Phi_{l}, \mathfrak{q}\right)$ are surjective.

(4.2) THEOREM. Let $A$ be a semilocal ring with at most one residue field isomorphic to $F_{2}$. If $\Phi$ is symplectic, assume further that $A=\boldsymbol{Z}\left[\left(A^{*}\right)^{2}\right]$ (this is automatic if $\left.2 \in A^{*}\right)$. Then $L(\Phi, A)=D(\Phi, A)$ and the maps $\theta_{l}$ of $\S 1$ are surjective. Moreover, if $\Phi$ and $A$ are as in (3.1), $\operatorname{St}(\Phi, A)$ is the universal central extension of $E(\Phi, A)$ and $L(\Phi, A) \approx H_{2}(E(\Phi, A), \boldsymbol{Z})$.

Note. Matsumoto [5] has shown the injectivity of $\theta_{l}$ when $A$ is a field. A paper of the author's now in preparation describes certain 
new identities satisfied by $\{$,$\} which imply this injective stability$ theorem for a radical ideal in a semilocal ring generated by its units.

The proof of (4.1) is based on the following decomposition of the group $\operatorname{St}(\Phi, q)$ when $q \subset \operatorname{rad} A$, similar to the Bruhat decomposition [2], [9] of the Chevalley groups.

In $\operatorname{St}(\Phi, q)$ let $\hat{U}(\Phi, q)$ be the subgroup generated by all $x_{\alpha}(q)$, $\alpha>0, q \in \mathfrak{q}$, and $\hat{O^{-}}(\Phi, \mathfrak{q})$ be the subgroup generated by all $x_{\alpha}(q)$, $\alpha<0, q \in \mathfrak{q}$.

(4.3) Theorem. The product map

$$
\hat{0}^{-}(\Phi, \mathfrak{q}) \times \hat{H}(\Phi, \mathfrak{q}) \times \hat{0}(\Phi, \mathfrak{q}) \stackrel{\psi}{\rightarrow} \operatorname{St}(\Phi, \mathfrak{q})
$$

is injective, and $L(\Phi, \mathfrak{q}) \cap \operatorname{im} \psi \subset \hat{H}(\Phi, \mathfrak{q})$. If $\psi$ is surjective, then $\mathfrak{q} \subset$ $\operatorname{rad} A$.

Conversely, suppose $\mathfrak{q} \subset \operatorname{rad} A$ and assume $A=Z\left[\left(A^{*}\right)^{2}\right]$ (resp. $\left.\boldsymbol{Z}\left[A^{*}\right]\right)$ if $\Phi$ is symplectic (resp. nonsymplectic). Then $\psi$ is surjective.

Using these theorems together with known properties [8], [9] of the pairing $\{$,$\} , one may derive quantitative information about the$ groups $L(\Phi, A)$ and, in particular, about $K_{2}(A)$. Some examples are:

Corollary. Let $m \in \boldsymbol{Z}, m>0, m \neq 0 \bmod 4$. Then $L(\Phi, \boldsymbol{Z} / m \boldsymbol{Z})=0$.

For $K_{2}$, this was proved by Milnor [6] using his computation of $K_{2}(\boldsymbol{Z})$ and results of Mennicke, Bass, Lazard, and Serre on the congruence subgroup problem. More generally:

Corollary. Let $\mathfrak{D}$ be a Dedekind domain of characteristic 0 , $0 \neq \mathfrak{p} \subset \mathfrak{O}$ a prime ideal which is unramified over $p \boldsymbol{Z}=\mathfrak{p} \cap \boldsymbol{Z}$. If $\operatorname{rk} \Phi=1$, assume that $\mathfrak{D} / \mathfrak{p} \neq F_{9}$. Then if $p$ is odd, $L\left(\Phi, \mathfrak{O} / \mathfrak{p}^{n}\right)=0$ for all $n \geqq 1$. Moreover, if $\Phi$ is nonsymplectic and $p=2, L\left(\Phi, \mathfrak{p}^{n-1} / \mathfrak{p}^{n}\right)$ is the product of at most $2^{8}-1$ cyclic groups of order 2 , where $\mathfrak{O} / \mathfrak{p}$ has cardinality $2^{s}$.

Corollary. The map $H_{2}\left(\mathrm{SL}\left(2, \boldsymbol{Z} / 2^{n} \boldsymbol{Z}\right), \boldsymbol{Z}\right) \rightarrow L\left(A_{1}, \boldsymbol{Z} / 2^{n} \boldsymbol{Z}\right)$ is surjective for $n=1,2$, but not for $n \geqq 3$.

Note. This corollary implies that $\{-1,-1\} \neq 0$ in $L\left(A_{1}, \boldsymbol{Z} / 4 \boldsymbol{Z}\right)$ (cf. [6]). This does not imply a similar result for $K_{2}(Z / 4 Z)$, since it is only known that the map $L\left(A_{1}, \boldsymbol{Z} / 4 \boldsymbol{Z}\right) \rightarrow K_{2}(\boldsymbol{Z} / 4 \boldsymbol{Z})$ is surjective.

\section{REFERENCES}

1. H. Bass and J. Tate, $K_{2}$ of global fields (in prep.).

2. N. Bourbaki, Groupes et algèbres de Lie, Fasc. 34, Chapitres 4, 5, 6, Actualités Sci. Indust., no. 1337, Hermann, Paris, 1968. MR 39 \#1590.

3. C. Chevalley, Certains schémas de groupes semi-simples, Séminaire Bourbaki 1960/61, fasc. 3, exposé 219, Secrétariat mathématique, Paris, 1961. MR 27 \#1339. 
4. M. Kervaire, Multiplicateurs de Schur et K-théorie, Essays on Topology and Related Topics, Memoires dédiés à Georges de Rham, Springer-Verlag, Berlin, 1970.

5. H. Matsumoto, Sur les sous-groupes arithmétiques des groupes semi-simples déployés, Ann. Sci. École Norm. Sup. (4) 2 (1969), 1-62. MR 39 \#1566.

6. J. Milnor, Algebraic K-theory, Van Nostrand Lecture Notes, Van Nostrand, Princeton, N. J. (to appear).

7. I. Schur, Über die Darstellungen der endlichen Gruppen durch gebrochene lineare Substitutionen, J. Reine Angew. Math. 127 (1904), 20-50; ibid. 132 (1907), 85-137.

8. R. Steinberg, Générateurs, rélations et revêtements de groupes algêbriques, Colloq. Théorie des Groupes Algébriques (Bruxelles, 1962), Librairie Universitaire, Louvain; Gauthier-Villars, Paris, 1962, pp. 113-127. MR 27 \#3638.

9. - Lectures on Chevalley groups, Lecture Notes, Yale University Math. Dept., New Haven, Conn., 1967.

Columbia University, New York, New York 10027 